\title{
Organic ice resists for 3D electron-beam processing: Instrumentation and operation
}

Tiddi, William; Elsukova, Anna; Beleggia, Marco; Han, Anpan

Published in:

Microelectronic Engineering

Link to article, DOI:

10.1016/j.mee.2018.01.021

Publication date:

2018

Document Version

Peer reviewed version

Link back to DTU Orbit

Citation (APA):

Tiddi, W., Elsukova, A., Beleggia, M., \& Han, A. (2018). Organic ice resists for 3D electron-beam processing: Instrumentation and operation. Microelectronic Engineering, 192, 38-43.

https://doi.org/10.1016/j.mee.2018.01.021

\section{General rights}

Copyright and moral rights for the publications made accessible in the public portal are retained by the authors and/or other copyright owners and it is a condition of accessing publications that users recognise and abide by the legal requirements associated with these rights.

- Users may download and print one copy of any publication from the public portal for the purpose of private study or research.

- You may not further distribute the material or use it for any profit-making activity or commercial gain

- You may freely distribute the URL identifying the publication in the public portal

If you believe that this document breaches copyright please contact us providing details, and we will remove access to the work immediately and investigate your claim 


\section{Organic ice resists for 3D electron-beam processing: instrumentation and operation}

William Tiddi, Anna Elsukova, Marco Beleggia, Anpan Han

DTU Danchip/Cen, Technical University of Denmark, Ørsteds Plads, Building 347, 2800 Kgs. Lyngby, Denmark.

Corresponding author: Anpan Han. Email: anph@dtu.dk

\section{Abstract}

Organic vapors condensed into thin layers of ice on the surface of a cold substrate are exposed with an electron beam to create resist patterns for lithography applications. The entire spin- and development-free lithography process requires a single custom instrument. We report the design, material choice, implementation and operation of this apparatus. It is based on a scanning electron microscope fitted with an electron beam control system that is normally used for electron beam lithography in a multi-user open-access laboratory. The microscope was also equipped with a gas injection system, a liquid nitrogen cooled cryostage, a temperature control system, and a load-lock. Three steps are required to initialize the apparatus for organic ice resist processing, and two steps are required to restore the apparatus for routine multi-user operations. Five steps are needed to create organic ice resist patterns. Finally, by stacking nanoscale patterns made in organic ice we created 3D structures using two complementary cyclic condense, expose and sublimate processes.

\section{Graphical abstract}



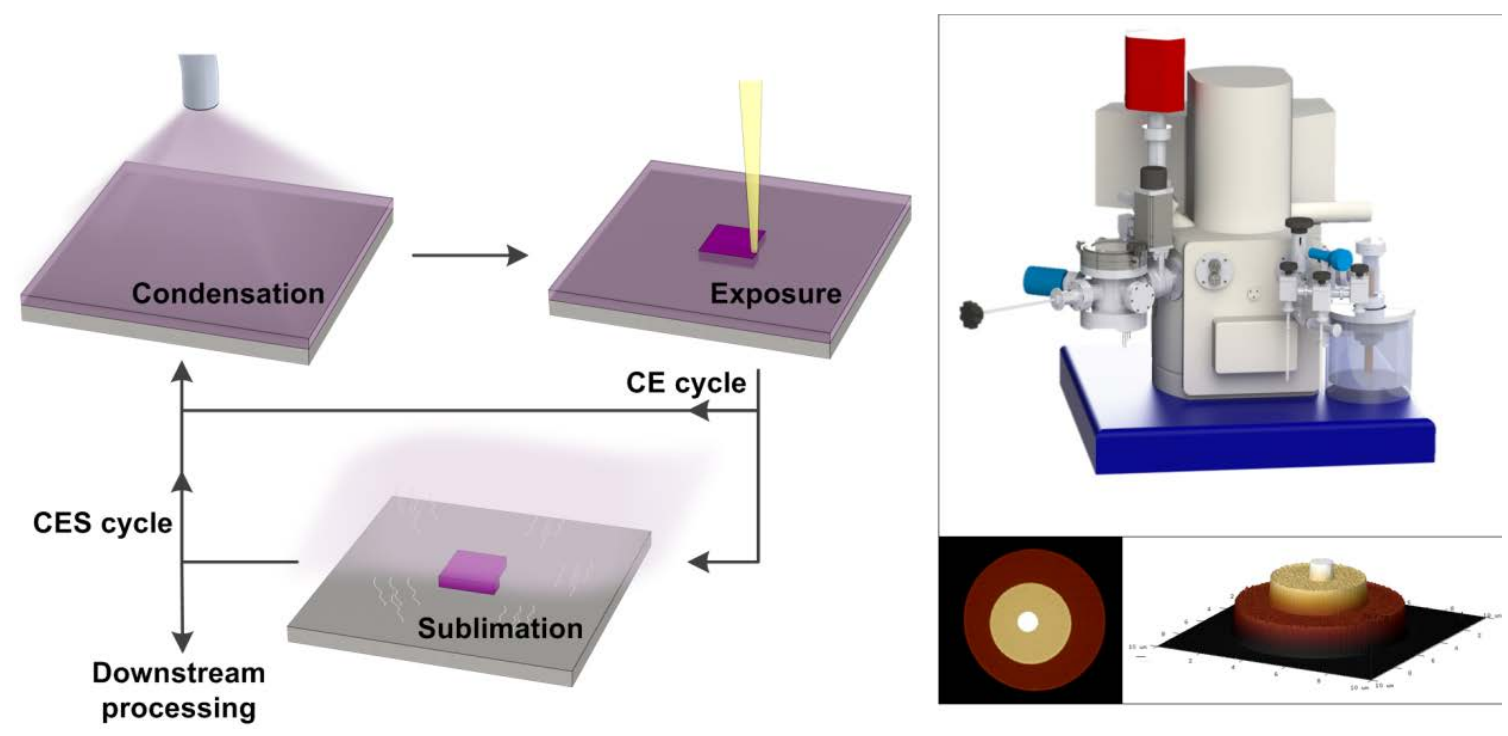

\section{Highlights}

- Spin- and development-free lithography process using condensed organic molecules at low temperatures.

- In-depth instrument design, realization and process flow.

- Two approaches for 3D multilayer lithography by stacking condensed organic ice layers.

Keywords: electron beam lithography, negative tone resist, organic ice resist, multilayer resist, 3D lithography

\section{Introduction}

Electron beam lithography (EBL) is used to create patterns for the fabrication of nanodevices. The EBL process for a negative-tone resist consist of four steps: i) resist spin-coating of the sample, ii) sample baking to remove the resist solvents, iii) exposing resist to a focused beam of electrons that locally modifies the resist chemistry, iv) immersing the sample with exposed resist 
into a developer that selectively dissolves the unexposed resist, while the exposed areas remain. Other complementary e-beam based patterning methods are focused electron beam induced deposition (FEBID) [1], ice lithography (IL) [2]-[4], and e-beam processing with organic ice resists (OIR) [5]. In FEBID, a precursor gas is injected near the sample held in high vacuum; the precursor gas molecules adsorb onto the sample surface, and the e-beam electrons react with them; as a result, a non-volatile deposit is formed. In IL, the sample is cooled down to $110 \mathrm{~K}$ so that it condenses injected water vapor to a layer of water ice coating the sample. Then, the ebeam is used to locally remove the ice to define patterns. These patterns in the water ice can then be transferred into metal patterns by an in-situ cryogenic metal deposition and "lift-off" process. Instead of using water ice, Balhke et al. showed that carbon dioxide ice was compatible with large area processing of organic thin-film semiconductors, which is challenging with established lithography methods [6]. In e-beam processing of OIR [5], organic vapors are frozen to ice layers on a cold sample, and the e-beam is used to expose the organic ice. Examples of organic vapors are isopropanol, nonane, anisole. After ice sublimation, a non-volatile product is found in the exposed areas. This allows OIR to be used as negative-tone EBL resists for making nanodevices. Other advantages of OIR are e.g. 3D lithography capabilities, patterning on very fragile non-planar samples, plasma etch selectivity comparable to photoresists, several orders of magnitude faster than FEBID, and the user is not exposed to any chemicals [5]. However, it is several orders of magnitude slower than EBL. In this report, we describe the apparatus for processing OIR, operation of the apparatus, and two complementary processing methods for the fabrication of 3D OIR structures.

\section{The apparatus}




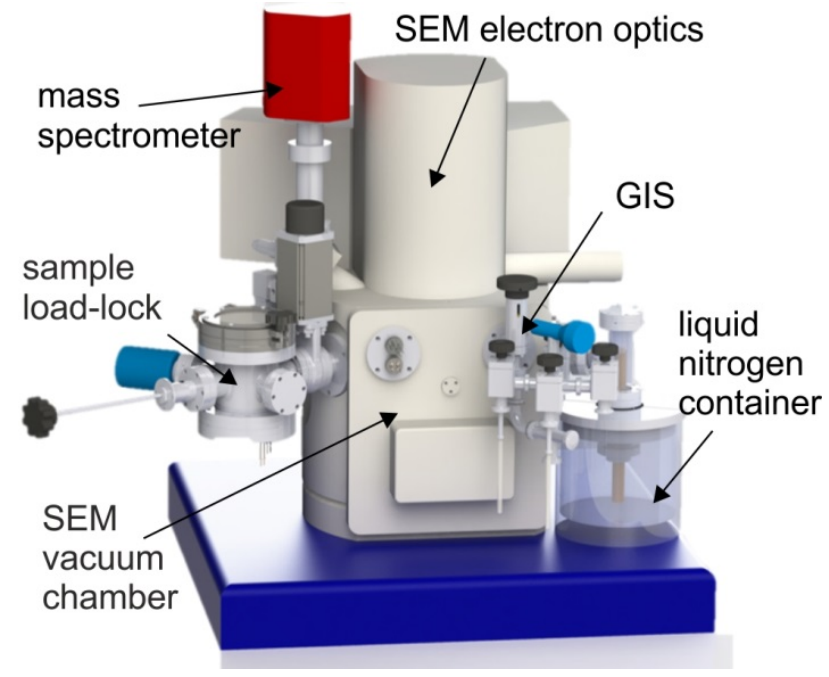

Figure 1. OIR instrument. The Zeiss SEM is modified with custom-made assemblies to accommodate the cryogenic operation and gas inlet needed to condense the organic vapors inside the chamber. Load-lock is added for rapid sample exchange and ice sublimation. The mass spectrometer monitors the SEM vacuum.

Our OIR instrument (Fig. 1), inspired by the ice lithography system [7], is based on a LEO SEM from Zeiss, which is equipped with an e-beam lithography system (ELPHY Quantum from Raith Gmbh, Germany). The SEM performance was evaluated using a gold on carbon sample, and the stage vibrations are about $30 \mathrm{~nm}$, which made the system only suitable for EBL patterning of structures larger than $100 \mathrm{~nm}$. The SEM base pressure is $5 \cdot 10^{-6} \mathrm{mbar}$. On the exterior of the OIR instrument, we added a load-lock chamber for sample transfer and warm-up. Also visible on the instrument exterior is the gas injection system (GIS), which is a manifold assembly consisting of a UHV leak valve and $3 \mathrm{HV}$ angle valves (VAT, Switzerland). When all valves are shut, a cell with fixed volume containing gas is formed. The gas pressure in the cell is measured using a convection enhanced Pirani gauge (Kurt J. Lesker, USA). For the GIS, all fittings and vacuum parts are of HV type. A residual gas analyzer (RGA) or mass spectrometer (Pfeiffer Vacuum, Germany) is installed to monitor the SEM vacuum quality. The liquid nitrogen (LN2) for cooling the cold 
finger and cryostage is kept in a standard 3 liter LN2 vacuum flask. The flask is placed on the SEM table, which is vibration insulated from the SEM vacuum chamber and electron optics.

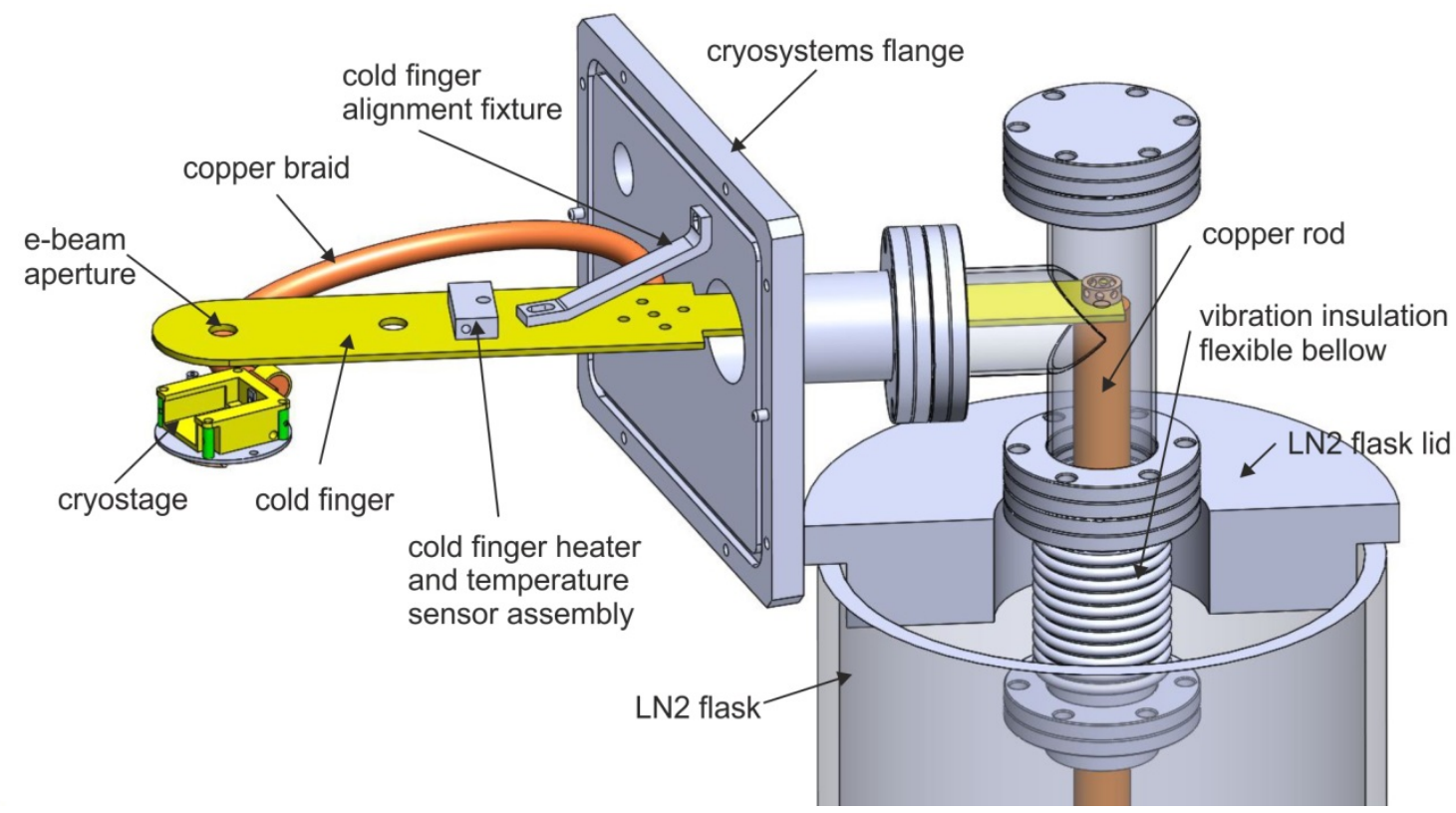

Figure 2. Cryosystems flange and assembly. The cold path starts from the copper rod that is immersed in liquid nitrogen in the dewar. The cold finger is attached to the vacuum end of the copper rod, and the cryostage is connected to the cold finger through a copper braid.

Inside the SEM chamber, we added a cold finger, a cryostage, and a stainless tube that is connected to the GIS, which is on the outside the SEM. The cold finger and cryostage is mounted onto the same vacuum flange (Fig. 2). The cold finger is $2.5-\mathrm{mm}$-thick, up to 50 -mm-wide and 340-mm-long. It is mechanically clamped to a copper rod feed-through, whose ambient end is immersed into LN2. For vibration and thermal insulation, the copper rod is silver-soldered onto a stainless steel flexible bellow. The cold finger is mechanically clamped to a flexible copper braid whose other end is clamped to the cryostage. Hence, the cold finger and the cryostage are cooled with the same LN2 source. The temperature increases along the cold path due to thermal losses. This arrangement is different from the ice lithography instrument, in which the cryostage 
and cold finger are mounted onto two separate flanges and cooled by separate LN2 sources, which enables separate temperature control. Our solution is compact and suits microscopes with fewer ports than the JEOL 7001F SEM used for IL [7]. The cold finger temperature can be adjusted with a 1/8 inch diameter cartridge heater (Firerod, Watlow, USA). The cryostage temperature is regulated independently with an additional heater. The temperature feedback control system is custom built using standard temperature control parts including a low noise linear power supply. Switched mode power supplies must be avoided, because they will generate excessive ripple electromagnetic fields, which would distort the SEM imaging and deteriorate lithography performance. The cold finger e-beam aperture is aligned with the electron optics, and the cold finger is then secured to the cryosystems flange with the alignment fixture made of thermal insulating stainless steel and PEEK washers. The cold finger is about 20 $\mathrm{K}$ colder than the cryostage, which allows efficient trapping of undesired condensable gases. Using the RGA, the water partial pressure is measured to $10^{-7}$ mbar which is 10 times higher than the IL instrument.
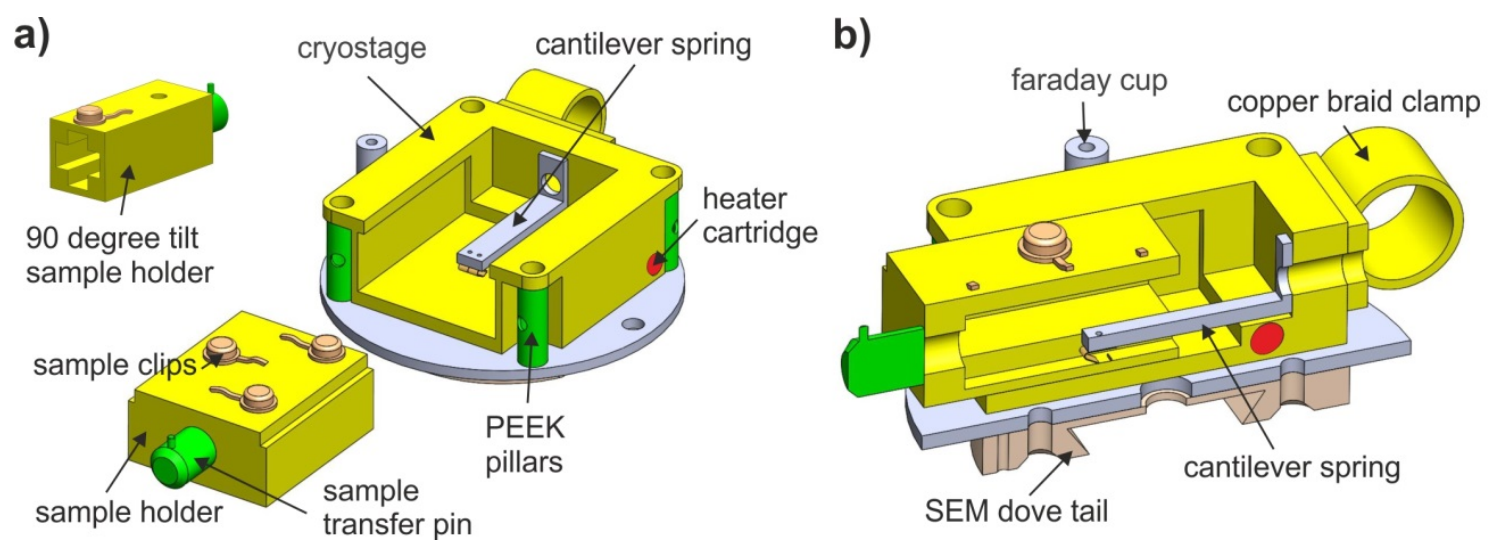

Figure 3. Cryostage and sample holders. 3D image of cryostage and the two different sample holders which can be used for sample transfer from the load-lock (a). Cut view of the sample holder partially inserted into the cryostage (b).

Thermal insulation of the IL instrument relied on three pins centering the cryostage. From experience, this design occasionally caused mechanical instability during sample transfer. To 
improve the mechanical stability, we adopted a different design: our cryostage is mounted onto the SEM stage dove tail, and it can be easily removed (Fig. 3). The cold part of the cryostage, which is connected to the copper braid via a clamping ring, is thermally insulated from the warm part of the cryostage via 4 pillars made in PEEK. The warm part of the cryostage is an adapted SEM dove tail. On the SEM dovetail is also mounted a Faraday cup for e-beam current measurement.

We carefully tested the performance of the cryosystems. Two hours after immersing the copper rod in LN2, the cold finger and cryostage cooled to $110 \mathrm{~K}$ and $130 \mathrm{~K}$, respectively. The lowest possible temperature of the cryostage is $120 \mathrm{~K}$, which is $10 \mathrm{~K}$ warmer than the IL instrument. The cooling rate is similar to the IL instrument. The PEEK pillar design proved to be significantly better regarding to mechanical stability and robustness during operation. The warm part of the cryostage is $9 \mathrm{~K}$ lower than ambient temperature, which did not have any impact on the SEM stage movement.

The sample transfer mechanism is of the bayonet type, which is similar to the IL instrument. The sample holder surface measures $25 \times 20 \mathrm{~mm}^{2}$, and up to 3 samples can be clamped onto it. The sample clips were standard SEM clips. Another dedicated sample holder allows 90 degree tilting of two samples. The sample holders have a hollow design that allows them to be held firmly down by a cantilever spring (Fig. 3b). The hollow design also reduces thermal mass for a faster sample holder cool down.

A sample load-lock was implemented to avoid condensation of ambient humidity onto a cold sample. It is mainly built with standard vacuum component. We decided to use UHV parts, because they are more mechanically stable than HV parts. The sample stage in the load-lock was 
custom made. A heater and a temperature control system are included for rapid ice sublimation and sample turn-over.

For the material selection, all cold parts were machined using copper and electroplated with gold for optimal heat conduction and oxidation prevention. Unless specified, all warm parts where either machined from aluminum, brass or stainless steel. We used non-magnetic aluminum and brass for parts in proximity to the e-beam, and stainless steel for its strength and thermal insulating properties. To compensate for thermal contraction of materials, and hence the risk of untightening after cool down, all screws and nuts were fastened with spring washers.

\section{OIR instrument operation}

\begin{tabular}{ccc} 
Step & Task & Time consumption (min) \\
\hline $1^{\dagger}$ & Mount cryosystems and GIS & 30 \\
$2^{+}$ & GIS preparation & 30 \\
$3^{\ddagger}$ & Initial cool down & 120 \\
4 & Sample loading and cool down & 100 \\
5 & OIR condensation & 10 \\
6 & SEM inspection (and alignment) & $10(+10)$ \\
7 & Exposure/Dose test & $0-80$ \\
8 & OIR sublimation and unload sample & 20 \\
$9^{\ddagger}$ & Systems heat up & 240 \\
$10^{+}$ & Unmounting OIR systems & 60
\end{tabular}

Table 1. Stepwise depiction of an OIR experimental session. Once the instrument is operational, steps 4 to 8 are repeated for each new sample. ${ }^{\dagger}$ OIR instrument installation procedures. ${ }^{\ddagger}$ Initialization and standby procedures.

We describe the detailed operation of the OIR instrument for making OIR patterns. All ten operation steps and corresponding time consumption are listed in Table 1. A video in the Supplementary Materials illustrates the OIR instrument operation step by step. 
Since our LEO SEM is a multi-user instrument that is normally used for SEM imaging and EBL, the first step for OIR lithography is to mount the cryosystems and the GIS, and the last step is to unmount them.

In step 2, a glass vial containing $5 \mathrm{ml}$ organic liquid is mounted onto the GIS. Then, all air inside the GIS is evacuated using a separate line that is connected to the load-lock backing pump. The valve between the GIS and load-lock pump is closed, and vapor pressure of the liquid is measured using the GIS pressure gauge until the vapor pressure stabilizes. We found the liquid in the vial sufficient for more than a week of intensive experiments.

In step 3, the SEM vacuum chamber is pumped down. Then the LN2 is poured into the flask, and the cryosystems cool down to operation temperatures. The cold finger also acts as a cryopump and condenses gasses in the SEM vacuum chamber. The pressure drops from $5 \cdot 10^{-6} \mathrm{mbar}$ to $1.7 \cdot 10^{-6} \mathrm{mbar}$.

In step 4, samples are clamped onto the sample holder and loaded into the load-lock. Samples that are challenging for spin-coating of EBL resists are perfectly suitable for OIR coating. We regularly patterned e.g. chips few $\mathrm{mm}$ in size, and containing fragile element such as 5-nm-thick suspended membranes. After pumping down to $5 \cdot 10^{-5} \mathrm{mbar}$, we open the gate valve between the SEM vacuum chamber and the load-lock, and the sample holder is transferred onto the cryostage. The sample cools down from room temperature to processing temperature after 80 min. To minimize sample surface contamination from the residual gasses in vacuum, the sample holder is moved to a position which is $1 \mathrm{~mm}$ under the cold finger. The cold-finger is much larger than the sample; due to the ballistic trajectory of gasses in high vacuum, the sample is well shielded from vacuum contaminants. 


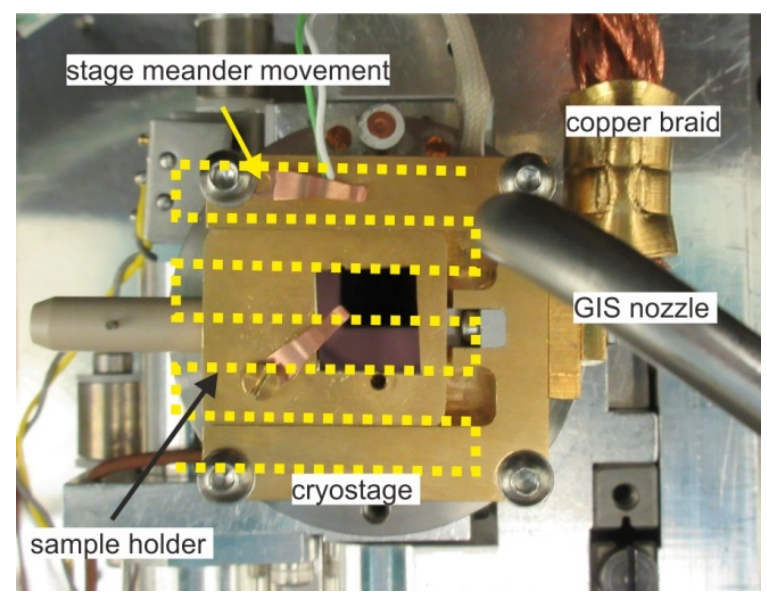

Figure 4. Cryostage movement under the GIS nozzle. For uniform condensation of OIR, the stage is moved in a meander path under the GIS nozzle. The nozzle is $5 \mathrm{~mm}$ above the sample surface.

In step 5, we condense an OIR layer onto the sample. The valve to the glass vial is shut, so that no additional vapor enters the GIS cell. We open the GIS leak valve, and the sample is moved 5 $\mathrm{mm}$ under the GIS nozzle. To cover the entire sample holder area, the stage is moved in a meander path with respect to the nozzle, such that the sample is coated with a uniform layer of OIR (Fig. 4).

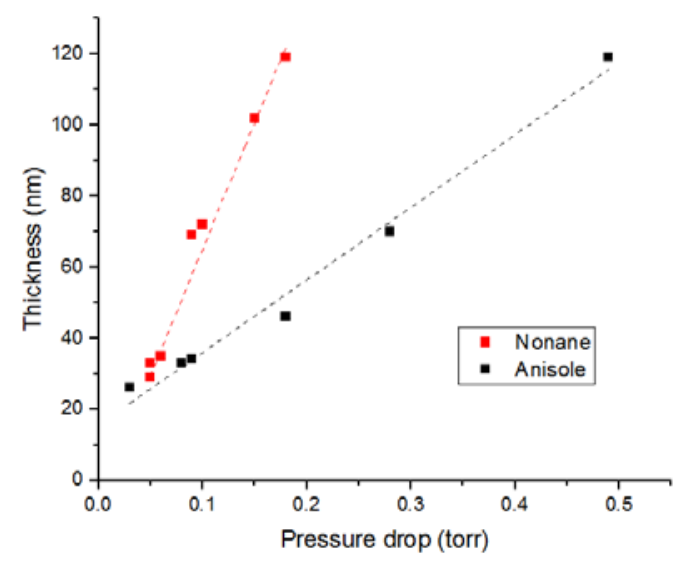

Figure 5. Calibration curve for OIR thickness. The thickness of exposed OIR measured by AFM is proportional to the pressure drop in the GIS cell. 
We monitor the GIS cell pressure while we introduce the vapor into the SEM for OIR condensation. To a first approximation, according to the ideal gas law, the pressure drop in the GIS cell $\left(\Delta \mathrm{P}_{\mathrm{GIS}}\right)$ is proportional to the amount of gas introduced into the $\mathrm{SEM}$, which is proportional to the condensed OIR thickness ( $\left.\Delta \mathrm{t}_{\mathrm{OIR}}\right)$. Assuming all gasses are condensed onto the cold sample, we have:

$$
\Delta t_{O I R}=\frac{V_{G I S} \mathrm{M}}{\rho \mathrm{ART}} \Delta P_{G I S}
$$

Here, $V_{G I S}$ is volume of the GIS cell, $M$ is the molar mass of the OIR, $\rho$ is the density of the ice, $A$ is the area where the ice is condensed, $\mathrm{R}$ is the gas constant, $\mathrm{T}$ is the room temperature. Thus, the thickness of the ice layer might be controlled by adjusting the leak valve and monitoring the

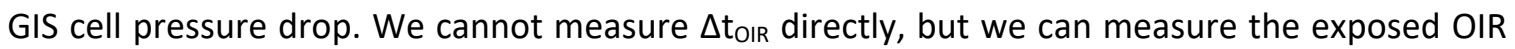
thickness after sublimation. As we expected, plotting the exposed OIR thickness and $\Delta \mathrm{P}_{\mathrm{GIS}}$, we see a linear dependence, and the slope is $700 \mathrm{~nm} /$ torr for nonane and $200 \mathrm{~nm} /$ torr for anisole (Fig. 5). The expected slopes according to the equation above for nonane and anisole are much greater (2300 and $1400 \mathrm{~nm} /$ torr, respectively). For this calculation, we used the liquid density of these materials as there are no tabulated values for their amorphous ice. The $V_{G I S}$ is $200 \mathrm{~cm}^{3}$, the area of the ice is $8.6 \mathrm{~cm}^{2}$. The mismatch between our calculations and experiments can be compensated by using a precursor-dependent correction factor. This correction factor accounts for approximations in the model, which might be e.g. compressibility factor of the vapors, precursor-specific sensitivity of the GIS gauge, differences between the as-condensed and exposed ice thickness, and losses of the vapor to the SEM chamber pump. 

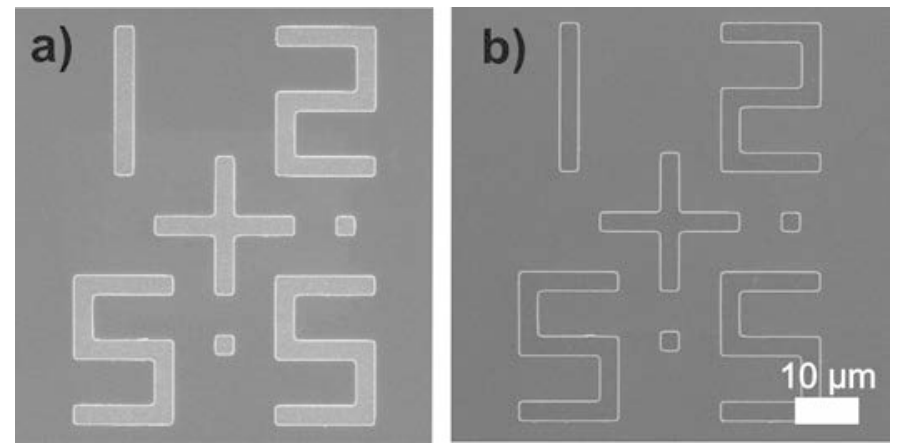

Figure 6. Alignment marks during OIR patterning. SEM images at $5 \mathrm{keV}$ showing Al alignment marks on silicon substrates, before (a) and after condensing 80-nm-thin nonane ice film (b). Imaging conditions were the same for (a) and (b).

In step 6, similar to EBL, the beam is optimized for e-beam exposure. If needed, an alignment procedure is performed. As with other EBL resists, the alignment marks remain visible in the SEM through the OIR layer (Fig. 6).

In step 7, we expose the OIR using the Raith EBL system. Similar to EBL, the first exposure is often the dose test. The dose can range between $1-30 \mathrm{mc} / \mathrm{cm}^{2}$, exposure current between 50 $\mathrm{pA}$ and $2 \mathrm{nA}$, and e-beam energy between 5 and $20 \mathrm{keV}$.
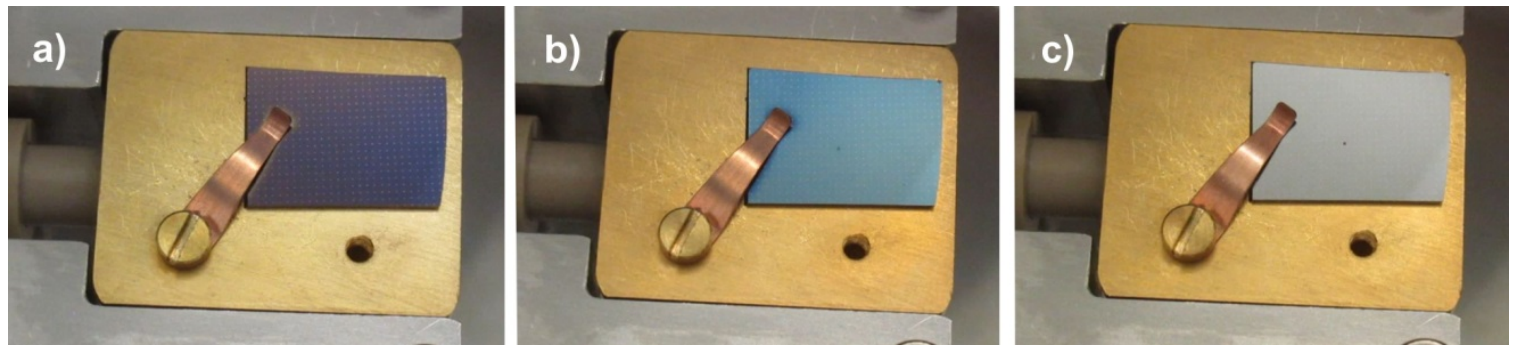

Figure 7. OIR sublimation process in the load-lock. The images show the same silicon sample on the gold plated sample holder inside the load-lock. The sublimation takes a few minutes. As the nominal OIR thickness decrease from 75 to $0 \mathrm{~nm}$, the sample color changes gradually from deep blue (a) to light blue (b) to gray (c). Note the uniformity of the colors, which indicate an OIR film with excellent thickness uniformity. 
In step 8, the OIR sublimation takes place after the exposure. The sample is transferred into the load-lock, and it is heated such that the OIR sublimates, which is clearly visible through the loadlock glass port (Fig. 7). The colors during the sublimation process also give an indication of the excellent thickness uniformity of the OIR layer. The load-lock stage is heated to room temperature, and the sublimated vapor is removed by the load-lock pumps.
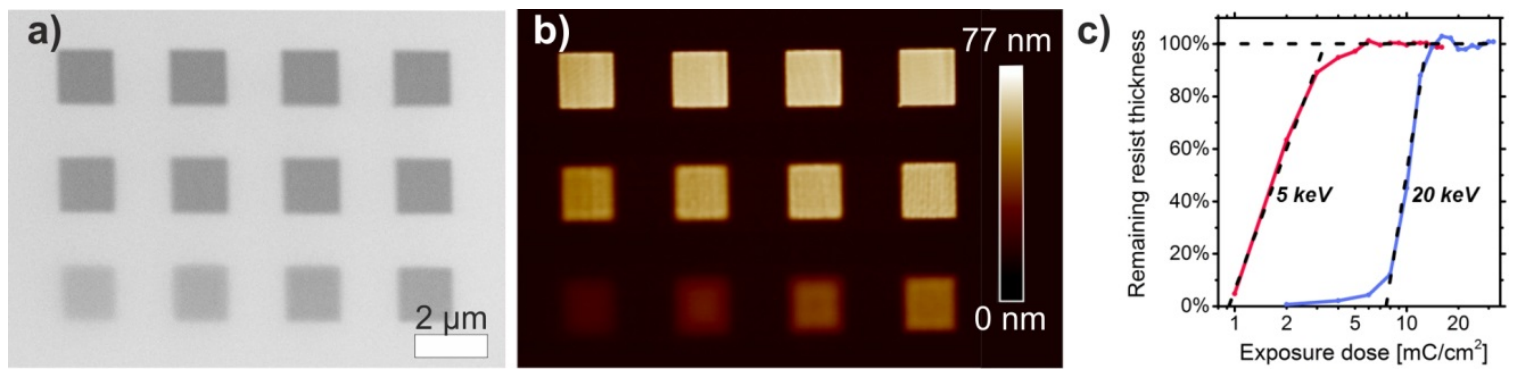

Figure 8. OIR dose test and contrast curve. SEM image of dose test after OIR sublimation (a). To examine the exposure quality, SEM images can be quickly taken in-situ. AFM analysis of dose test (b), and contrast curve for nonane for two different e-beam energies (c).

After the sublimation, the sample can be optionally transferred into the SEM vacuum chamber, and the critical dose inspected in SEM by imaging. SEM images give an indication of the exposure quality and an estimate of the critical dose (Fig. 8a). For an accurate measurement of the critical dose, AFM analysis (Fig. 8b) is performed to create the contrast curve (Fig. 8c).

In steps 9 and 10, after the OIR experiments, we empty the LN2 flask, and the cryosystems are heated to room temperature. Then, the OIR cryosystems and GIS can be removed from the LEO SEM such the instrument can be used for imaging and EBL.

\section{D OIR processing}




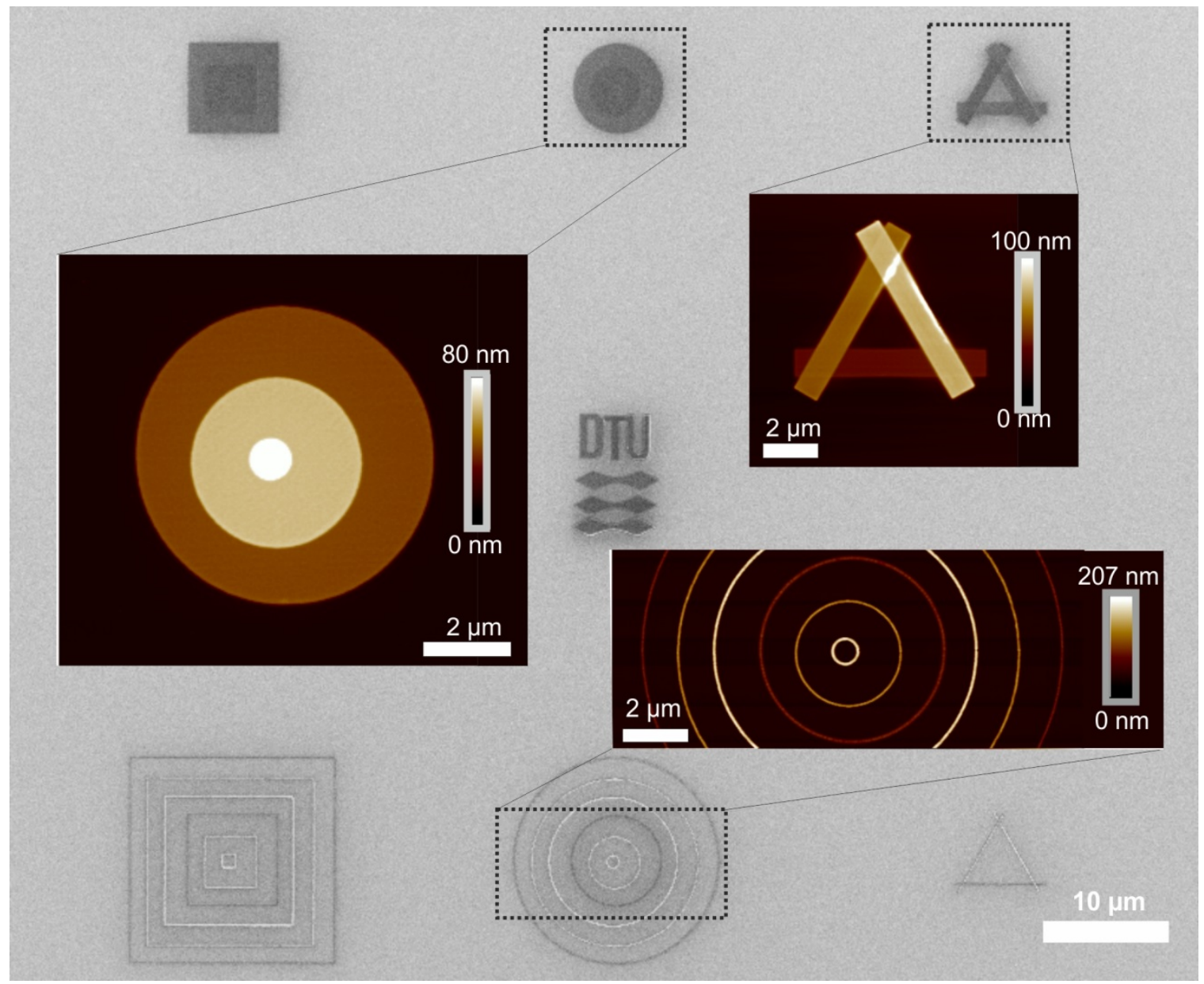

Figure 9. 3D OIR structures using 3 layers of OIR. Structures were made using a series of three CE cycles. The background image was taken with the SEM, and the magnified images were taken with the AFM. Each OIR layer is approximately 45-nm-thick. The narrow lines have a total thickness of $135 \mathrm{~nm}$, while the larger areas were underexposed, which resulted in a total thickness of $80 \mathrm{~nm}$.

Using a layer-by-layer approach, we can efficiently pattern OIR in 3D. Similar to EBL, the dose required to expose OIR is independent of the resist thickness. The critical dose did not change significantly between 30 and $600 \mathrm{~nm}$ OIR layers [5]. We use two methods to obtain 3D OIR structures, either by condense-expose (CE) cycles, or by condense-expose-sublimation (CES) cycles. 
In the CE process, a layer of OIR is condensed onto the sample and the e-beam exposure follows. After the exposure, another layer of OIR is condensed and exposed. The total ice thickness increases after each cycle. Once the sample is heated after the final cycle, and the unexposed ice sublimates, the exposed areas form layered 3D structures. Each structure has the cumulative ice thickness at the cycle when it was exposed. In other words, the user can place an exposure at any condensation step, according to the desired feature thickness. We show 3D structures with a CE-CE-CE process (Fig. 9). The smallest features were $100 \mathrm{~nm}$. Each OIR layer is 45-nmthick.

Compared to the CE cycle, a CES cycle contains an additional sublimation step between the exposure and the next condensation. To monitor the sublimation process, we move and heat the sample in the load-lock stage. The cryostage heater can also be used for this purpose. All unexposed ice is removed, leaving only the previously patterned features on the surface. This allows performing layer-to-layer alignment and multi-patterning to increase pattern density. Unlike in the CE cycle, the unexposed ice thickness doesn't increase after each cycle due to the sublimation step. Overlaps with exposed OIR patterns from previous layers will result in features with increased height.

For 3D structures, CES enable different chemistries that are not compatible with CE process, e.g. in the case where two different unexposed OIR layers would chemically react or present adhesion issues. In the CES process the two unexposed ices would never come into contact. A sequence of combined CE and CES cycles can be used to allow desired multilayer designs. The GIS allows two or more vials to be mounted simultaneously, and this allows switching between different OIRs. 


\section{Conclusions and future work}

We report a custom OIR apparatus that is based on a SEM normally used for EBL in a multi-user open access laboratory. Dedicated to the LEO SEM from Zeiss, a new cryosystems and cryostage design and implementation are presented. 3 hours are required to initialize the SEM for OIR processing, and 4 hours warm up a cold instrument to room temperature. Sample turnover requires about 2 hours excluding the exposure time.

Using this custom instrument, we demonstrated successful 3D OIR patterning with $100 \mathrm{~nm}$ minimal feature size, and $135 \mathrm{~nm}$ total pattern thickness. The minimal feature size is not limited by the physical and chemical characteristics of the OIR, but by instrumental limitations of the multi-user SEM [5].

For more efficient OIR processing, there are 3 major instrumentation tasks that lay ahead. First, we need to decrease the sample cool down time to less than $10 \mathrm{~min}$, which would shorten the sample turn over time down to $50 \mathrm{~min}$. This could be achieved by improving the cryostage and sample holder design. Second, for 3D OIR patterns with hundreds or thousands of layers, the GIS must be automated and integrated seamlessly with the lithography system. Third, while our apparatus is sufficiently cold for freezing nonane and anisole, it will not freeze other sustainable chemicals such as ethanol. A completely new cryostage design is needed for sustainable chemistry.

\section{Acknowledgements}

The authors would like to acknowledge J. E. Jørgensen, M. Nimb, T. Feld and S. M. B. Petersen for providing custom parts in the experimental setup. The authors thank for their contribution, assistance and valuable inputs A. Gregersen (for the electronics setup), R. Cork (vacuum setup), J. 
Michael-Lindhard (SEM instrument). We also acknowledge financial support from the VILLUM foundation. The authors declare no competing interests.

\section{References}

[1] C. W. Hagen, "The future of focused electron beam-induced processing," Appl. Phys. A Mater. Sci. Process., vol. 117, no. 4, pp. 1599-1605, 2014.

[2] A. Han, A. Kuan, J. Golovchenko, and D. Branton, "Nanopatterning on nonplanar and fragile substrates with ice resists," Nano Lett., vol. 12, no. 2, pp. 1018-1021, 2012.

[3] G. M. King, G. Schurmann, D. Branton, and J. Golovchenko, "Nanometer Patterning with Ice," Nano Lett., vol. 5, pp. 1157-1160, 2005.

[4] A. Han, D. Vlassarev, J. Wang, J. A. Golovchenko, and D. Branton, "Ice lithography for nanodevices," Nano Lett., vol. 10, no. 12, pp. 5056-5059, 2010.

[5] W. Tiddi, A. Elsukova, H. T. Le, P. Liu, M. Beleggia, and A. Han, "Organic Ice Resists," Nano Lett., p. acs.nanolett.7b04190, Nov. 2017.

[6] M. E. Bahlke, H. A. Mendoza, D. T. Ashall, A. S. Yin, and M. A. Baldo, "Dry Lithography of Large-Area, Thin-Film Organic Semiconductors Using Frozen $\mathrm{CO}_{2}$ Resists," Adv. Mater., vol. 24, no. 46, pp. 6136-6140, Dec. 2012.

[7] A. Han, J. Chervinsky, D. Branton, and J. A. Golovchenko, "An ice lithography instrument," Rev. Sci. Instrum., vol. 82, no. 6, 2011. 